\title{
Apoptosis of HeLa cells induced by a new targeting photosensitizer-based PDT via a mitochondrial pathway and ER stress
}

This article was published in the following Dove Press journal:

OncoTargets and Therapy

7 April 2015

Number of times this article has been viewed

\section{Donghong $\mathrm{Li}^{1}$ \\ Lei $\mathrm{Li}^{2}$ \\ Pengxi Li' \\ $\mathrm{Yi} \mathrm{Li}^{3}$ \\ Xiangyun Chen'}

'State Key Laboratory of Trauma, Burn and Combined Injury, The Second Department of Research Institute of Surgery, ${ }^{2}$ The First Department of Research Institute of Surgery, ${ }^{3}$ Cancer Center, Daping Hospital, Third Military Medical University, Chongqing, People's Republic of China

Correspondence: Donghong $\mathrm{Li}$ The Second Department of Research Institute of Surgery, Daping Hospital, Third Military Medical University, Chongqing 400042, People's Republic of China

Tel +86 2368757423

Fax +862368757421

Email lqs_cq@aliyun.com
Abstract: Photodynamic therapy (PDT) is emerging as a viable treatment for many cancers. To decrease the cutaneous photosensitivity induced by PDT, many attempts have been made to search for a targeting photosensitizer; however, few reports describe the molecular mechanism of PDT mediated by this type of targeting photosensitizer. The present study aimed to investigate the molecular mechanism of PDT induced by a new targeting photosensitizer (PS I), reported previously by us, on HeLa cells. Apoptosis is the primary mode of HeLa cell death in our system, and apoptosis occurs in a manner dependent on concentration, irradiation dose, and drug-light intervals. After endocytosis mediated by the folate receptor, PS I was primarily localized to the mitochondria and the endoplasmic reticulum (ER) of HeLa cells. PS I PDT resulted in rapid increases in intracellular reactive oxygen species (ROS) production and $\mathrm{Ca}^{2+}$ concentration, both of which reached a peak nearly simultaneously at 15 minutes, followed by the loss of mitochondrial membrane potential at 30 minutes, release of cytochrome $\mathrm{c}$ from mitochondria into the cytoplasm, downregulation of Bcl-2 expression, and upregulation of Bax expression. Meanwhile, activation of caspase-3, -9, and -12, as well as induction of C/EBP homologous protein (CHOP) and glucose-regulated protein (GRP78), in HeLa cells after PS I PDT was also detected. These results suggest that apoptosis of HeLa cells induced by PS I PDT is not only triggered by ROS but is also regulated by $\mathrm{Ca}^{2+}$ overload. Mitochondria and the ER serve as the subcellular targets of PS I PDT, the effective activation of which is responsible for PS I PDT-induced apoptosis in HeLa cells.

Keywords: folate-PEG-chlorin, folate receptor positive cells, cell death model, mechanism

\section{Introduction}

Photodynamic therapy (PDT) is emerging as a viable treatment option for many cancers. PDT is based on light-induced activation of a photosensitizer, which results in subsequent in situ production of reactive oxygen species (ROS), the latter directly destroying the cells that accumulate in the photosensitizer.

Despite the promising effect of PDT in the treatment of oncologic disease becoming increasingly clear, there is also growing awareness of its side effects. The long-lasting cutaneous photosensitivity after irradiation is a major side effect that severely impacts the applicability of PDT. To optimize PDT as a useful therapeutic option for malignant tumors, a number of attempts have been made to improve photosensitizer targeting by conjugating the photosensitizer to tumor-specific molecules, such as epidermal growth factor, monoclonal antibodies, carrier proteins, carbohydrates, and hydrophilic polymers. ${ }^{2-6}$

Because the folate receptor is overexpressed in human epithelial cancer cells but is absent from most normal cells and because folic acid can specifically bind to the folate receptor with high affinity, ${ }^{7}$ we previously synthesized a targeting photosensitizer, PS I, by conjugating folic acid with chlorin, an analog of meta-tetrahydroxyphenylchlorin 
(mTHPC), using polyethylene glycol (PEG) as the linker (Figure 1A). Human cervical carcinoma HeLa cells strongly express the folate receptor, which has been confirmed by our previous study ${ }^{8}$ and another report; ${ }^{9}$ therefore, the HeLa cell line was used as one of the test cell lines to investigate the targeting of PS I in our studies. We found that this new photosensitizer targeted HeLa cells much more effectively than the original, chlorin, and its water solubility was significantly improved. ${ }^{8}$

Although many studies generally focused on the development of targeting photosensitizers have been reported, the mechanism of cell death induced by these targeting photosensitizers remains to be elucidated. It is well known that the mechanism of PDT-mediated cell death is dependent not only on the cell line but also on the properties of the photosensitizer used. ${ }^{10}$ The primary aims of this study are to clarify the death pathway induced by PS I PDT in HeLa cells and to determine whether the structural modifications in PS I, especially the introduction of macromolecules (such as PEG) and tumor-targeting compounds (in this case, folic acid), could affect its cell death mechanism. To answer these questions, the production of ROS, 1) the changes in intracellular $\mathrm{Ca}^{2+}$ concentration $\left(\left[\mathrm{Ca}^{2+}\right]_{\mathrm{i}}\right)$ and mitochondrial membrane potential (MMP), 2) cytochrome c release, 3) expression levels of B cell lymphoma-2 (Bcl-2), Bcl-2 Associated X Protein (BAX), CCAAT/enhancer-binding protein (c/EBP) homologous protein (CHOP), and glucose-regulated protein (GRP78), and 4) the activation of caspase-3, -9 , and -12 were investigated during the 48-hour period post-PDT in this study.

\section{Materials and methods \\ Chemicals and antibodies}

Cell culture materials were purchased from Costar (Dutscher, Brumath, France). Fetal bovine serum (FBS), penicillin,
A
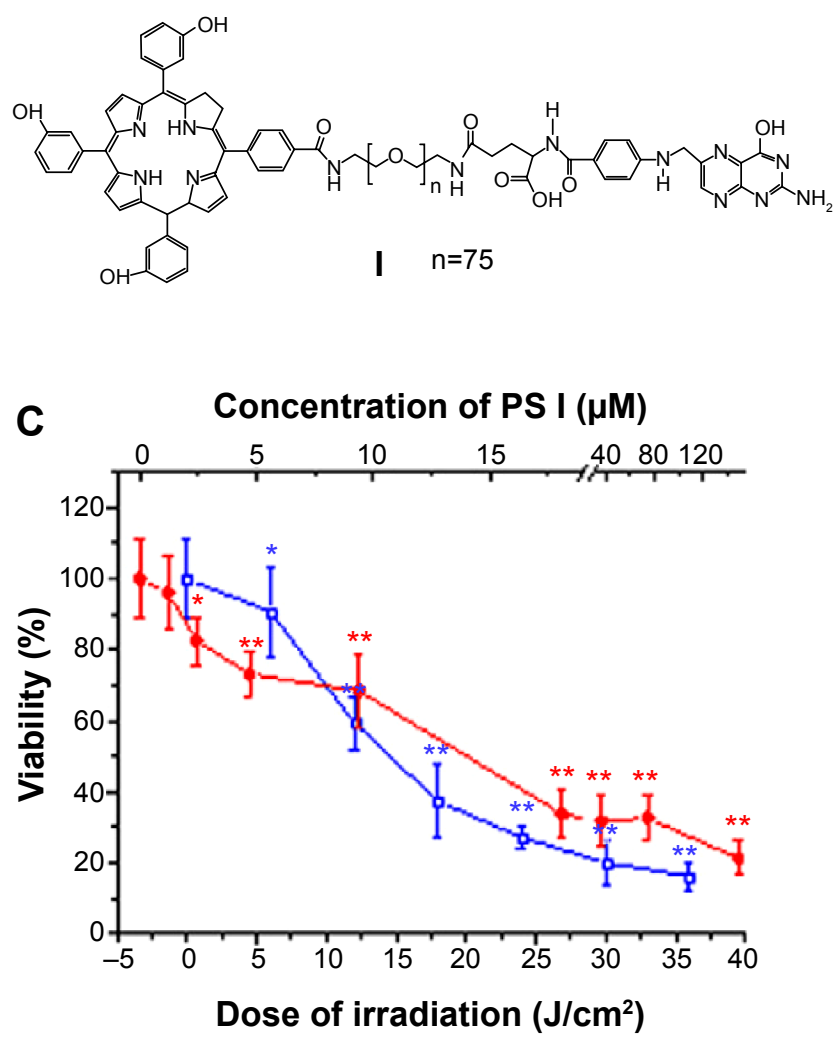

B

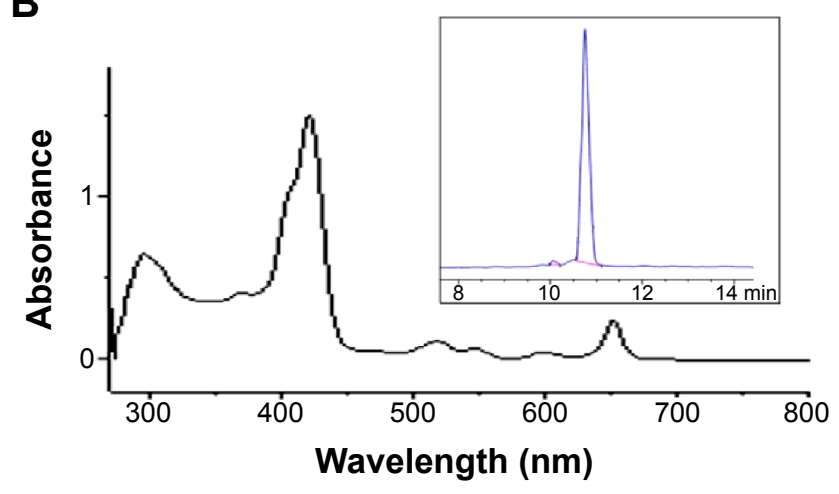

D

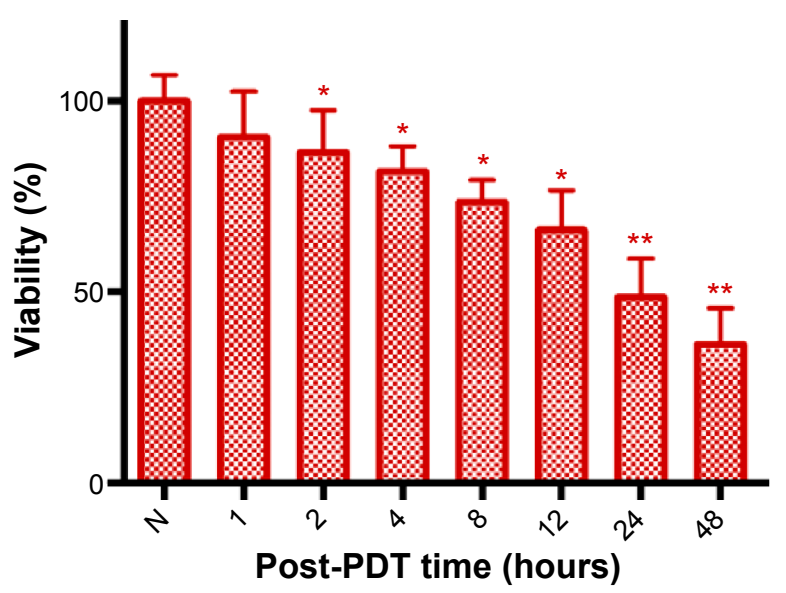

Figure I The characteristics and cytotoxicity of PS I.

Notes: (A) Molecular structure of PS I. (B) The absorption spectrum; insert: HPLC spectrum. (C) Irradiation dose-dependent and concentration-dependent cytotoxicity. HeLa cells were treated with I5.2 $\mu$ M of PS I for 24 hours, then irradiated with the indicated dose (ranging from $0 \mathrm{~J} / \mathrm{cm}^{2}$ to $\left.36 \mathrm{~J} / \mathrm{cm}^{2}\right)(-\square-$, blue line); or cells were treated with the indicated concentration of PS I (ranging from $0 \mu \mathrm{M}$ to $147 \mu \mathrm{M})$ for 24 hours and then irradiated with $18 \mathrm{~J} / \mathrm{cm}^{2}$ of red light (- $\bullet-$, red line). Cell viability was assessed via MTT assay at 24 hours post-PDT. The samples treated with I5.2 $\mu \mathrm{M}$ of PS I without irradiation and irradiation with red light in the absence of PS I served as controls. (D) Post-PDT time-dependent cytotoxicity. Cells were treated with $15.2 \mu \mathrm{M}$ of PS I for 12 hours, followed by $18 \mathrm{~J} / \mathrm{cm}^{2}$ of red light. Cell viability was measured at the indicated times post-PDT. The cells without any treatment served as the control $(\mathrm{N})$. Data represent means \pm SDs $(n=3)$. $* P<0.05$ vs control, $* * P<0.01$ vs control.

Abbreviations: HPLC, high-performance liquid chromatography; MTT, methylthiazolyldiphenyl tetrazolium bromide; PDT, photodynamic therapy; PS I, photosensitizer I; $\mathrm{SD}$, standard deviation. 
streptomycin, Dulbecco's Modified Eagle's Medium (DMEM), and Dulbecco's phosphate buffered saline (DPBS) were purchased from Hyclone (South Logan, UT, USA). Trypsin, rhodamine 123 (R123), methylthiazolyldiphenyl tetrazolium bromide (MTT), 4',6-diamidino-2-phenylindole (DAPI), dimethyl sulfoxide (DMSO), and fluorescein isothiocyanate (FITC)-conjugated propidium iodide (PI) were purchased from Sigma-Aldrich. Annexin V-PI Apoptosis Detection Kit was purchased from BD Biosciences (San Jose, CA, USA). MitoTracker Green FM, LysoTracker Yellow HCK-123, ER-Tracker Blue-White DPX, 2',7'dichlorodihydrofluorescein diacetate ( $\left.\mathrm{H}_{2} \mathrm{DCFDA}\right)$, and fluo-4-(acetoxymethyl) ester (Fluo-4-AM) were obtained from Molecular Probes (Invitrogen, Carlsbad, CA, USA). Antibodies against cytochrome c, caspase-3, caspase-9, caspase-12, Bcl-2, Bax, CHOP, GRP78, and glyceraldehyde3-phosphate dehydrogenase were purchased from Abcam (Cambridge, UK).

\section{Cell culture}

The human cervical carcinoma HeLa cell line was obtained from the Chinese Academy of Sciences' Shanghai Cell Library, cultured in DMEM supplemented with 10\% FBS, penicillin $(100 \mu \mathrm{g} / \mathrm{mL})$, and streptomycin $(100 \mu \mathrm{g} / \mathrm{mL})$ under $5 \% \mathrm{CO}_{2}$ at $37^{\circ} \mathrm{C}$ in a humidified incubator, and routinely subcultured every 3 days.

\section{PDT protocol}

The novel targeting photosensitizer I (PS I), with a maximum absorption peak at $650 \mathrm{~nm}$ and a purity $>98 \%$ (Figure 1B), was synthesized in our laboratory. A stock solution $(1 \mathrm{mg} / \mathrm{mL})$ was prepared in FBS-free DMEM and stored covered in aluminum foil at $4^{\circ} \mathrm{C}$. The cultured cells were incubated with the indicated concentrations of PS I for the indicated times at $37^{\circ} \mathrm{C}$ in the dark. Subsequently, the cells were washed with DPBS three times, and the photosensitized cells were exposed to 600-700 $\mathrm{nm}$ red light generated by a KDH150B red-light therapy instrument (Kedian Co, Beijing, China) to activate PS I. During irradiation, the temperature never exceeded $25^{\circ} \mathrm{C} \pm 2^{\circ} \mathrm{C}$.

\section{Cytotoxicity evaluation}

Cell viability was measured using the MTT assay. HeLa cells at a density of $5 \times 10^{4}$ cells per well were incubated with PS I at $37^{\circ} \mathrm{C}$ in detachable 96 -well culture plates in the dark and then exposed to red light. After incubation for 24 hours, the cells were incubated in $0.5 \mathrm{mg} / \mathrm{mL}$ MTT solution for 4 hours at $37^{\circ} \mathrm{C}$, and the resulting insoluble purple formazan crystals were dissolved in DMSO. The absorbance at $492 \mathrm{~nm}$ was measured using a microplate reader model 450 (Bio-Rad, USA). Cell viability was proportional to the $\mathrm{A}_{492}$ value and was expressed as a percentage of the cell viability of control cells, and the cell viability in the control group (irradiation dose was $0 \mathrm{~J} / \mathrm{cm}^{2}$ ) represented the dark cytotoxicity. For each concentration or irradiation dose, three trials were performed.

\section{Detection of cytoskeletal and DNA damage}

HeLa cells were inoculated in $3 \mathrm{~cm}$ plates at a density of $1 \times 10^{5}$ cells per plate. After incubation for 10 hours in $15.2 \mu \mathrm{M}$ of PS I, cells were stained with FITC-phalloidin and DAPI, then irradiated with $18 \mathrm{~J} / \mathrm{cm}^{2}$ of red light. The untreated cells were used as control. Twelve hours after irradiation, cells were fixed with $4 \%$ paraformaldehyde for 10 minutes and permeabilized with $0.1 \%$ Triton X-100. After washing with DPBS, the cells were incubated in FITC-phalloidin for 60 minutes, followed by incubation in DAPI for 10 minutes. Samples were mounted under glass cover slides and observed under a laser confocal microscope (TCS SP5, Leica). ${ }^{11}$

\section{Assessment of apoptosis in HeLa cells}

HeLa cells were inoculated in $3 \mathrm{~cm}$ plates at a density of $5 \times 10^{5}$ cells per plate. At 24 hours or the indicated time post-PDT, HeLa cells were harvested and double-stained with Annexin V and PI using an Annexin V-PI apoptosis detection kit, according to the manufacturer's instructions. Stained cells were analyzed using a FACSCalibur flow cytometer (BD Biosciences). At least 10,000 events were collected for each sample.

\section{Subcellular localization of photosensitizer}

During the final 30 minutes of PS I incubation, cells were coloaded with ER-Tracker ${ }^{\text {TM }}$ Blue-White DPX, MitoTracker ${ }^{\circledR}$ Green FM, and LysoTracker ${ }^{\circledR}$ Yellow-HCK-123 at a final concentration of $500 \mathrm{nM}$ to label the endoplasmic reticulum (ER), mitochondria, and lysosomes, respectively. Doublestained cells were observed using a laser confocal microscope (TCS SP5, Leica). Organelle probes were excited using a $488 \mathrm{~nm}$ argon laser (MitoTracker ${ }^{\circledR}$ and LysoTracker ${ }^{\circledR}$ ) and a $405 \mathrm{~nm}$ diode laser (ER-Tracker). PS I was excited using a helium/neon laser at $488 \mathrm{~nm}$.

\section{Quantification of intracellular ROS levels}

Briefly, after incubation in $15.2 \mu \mathrm{M}$ of PS I for 12 hours, HeLa cells were incubated in $10 \mu \mathrm{M}$ of $\mathrm{H}_{2}$ DCFDA in culture medium 
lacking phenol red for 10 minutes and irradiated, followed immediately by continuous confocal scanning at an excitation wavelength of $488 \mathrm{~nm}$ with a 510-530 $\mathrm{nm}$ bandpass barrier filter to measure the change in fluorescence intensity.

\section{Measurement of MMP}

Briefly, PS I-treated HeLa cells were loaded with $2 \mu \mathrm{M}$ of R123 for 30 minutes. After gentle rinsing with DPBS, stained cells were irradiated and immediately monitored via continuous confocal scanning using the same filter settings as used for $\mathrm{H}_{2}$ DCFDA.

\section{Measurement of intracellular $\mathrm{Ca}^{2+}$ levels}

In brief, the photosensitized cells were incubated in $2 \mu \mathrm{M}$ of Fluo-4-AM in $\mathrm{Ca}^{2+}$-free medium ( $2 \mathrm{mM}$ of ethylene glycol tetraacetic acid was added to chelate any residual $\mathrm{Ca}^{2+}$ in the medium) for 30 minutes and irradiated. The stained cells were immediately observed via continuous confocal scanning using the same filter settings as used for ROS.

\section{Western blot assay}

HeLa cells were lysed using radio-immunoprecipitation assay (RIPA) buffer 24 hours after PDT. Equivalent amounts of proteins were electrophoresed on $10 \%$ polyacrylamide gels and transferred to polyvinylidene fluoride membrane. After blocking with 5\% skim milk for 1 hour, primary antibodies were bound overnight at $4^{\circ} \mathrm{C}$. Each membrane was probed with horseradish peroxidase-labeled goat anti-rabbit IgG antibody for 1.5 hours. The grayscale image of each labeled protein band was analyzed using Quantity One software (version 4.4.0; Bio-Rad).

\section{Statistical analysis}

The data are presented as means \pm standard deviation. Student's $t$-test was used for statistical analysis. The difference was considered statistically significant when $P<0.05$. SPSS software was used.

\section{Results}

\section{Photodynamic activity of PS I in HeLa cells}

The chemical structure, absorption spectrum, and high-performance liquid chromatography spectrum of PS I are shown in Figure 1A and B. As shown in Figure 1C, when the irradiation dose was zero, the viability of HeLa cells was $100 \%$, which indicated that the dark cytotoxicity of PS I to HeLa cells was very low. Similarly, the irradiation alone (the concentration of PS I was zero) was noncytotoxic to HeLa cells. The cytotoxicity induced by PS I PDT positively correlated with the irradiation dose (Figure $1 \mathrm{C}$, blue line, $R=0.962 ; P=0.001$ ) and the concentration of PS I (Figure $1 \mathrm{C}$, red line, $R=0.763$; $P=0.017)$. Meanwhile, cytotoxicity also occurred in a timedependent manner (Figure 1D). Cell viability was significantly reduced after 2 hours $(P<0.05)$ and $4-48$ hours $(P<0.01)$ of incubation in $15.2 \mu \mathrm{M}$ of PS I with PDT.

\section{Changes in F-actin and DNA structure following PDT}

To investigate the PDT effects induced by PS I on the cytoskeleton and DNA structure, cells were stained using FITC-PI and DAPI to visualize F-actin and DNA, respectively. In the control group (N), a network of F-actin filaments extending throughout the cell and nuclei displaying a regular, oval morphology could be observed. However, following PS I PDT, the fluorescence originating from the labeled filaments became weaker, and the nuclei became more circular and smaller (Figure 2A), indicating that after PDT, the cytoskeleton of HeLa cells lost its F-actin structure.

\section{Apoptosis and necrosis of HeLa cells induced by PS I PDT}

The percentage of apoptotic and necrotic cells was quantified via Annexin V and PI double staining. Compared to the control group, the numbers of apoptotic and necrotic cells just slightly increased in both the irradiation-alone group and the PS I-without-light group, but the number significantly increased in the PTD group, especially the number of apoptotic cells (Figure 2B). As the post-PDT time progressed, the number of apoptotic cells clearly increased (Figure 2C). Meanwhile, an irradiation dose-dependent (Figure 2D, red lines) and a concentration-dependent (Figure 2D, blue lines) apoptotic effect was observed. The quantities of both apoptotic and necrotic cells positively correlated with the irradiation dose (apoptosis $R_{\mathrm{a}}=0.966$; necrosis $R_{\mathrm{n}}=0.989$ ) and the concentration of PS I (apoptosis $R_{\mathrm{a}}=0.996$; necrosis $\left.R_{\mathrm{n}}=0.983\right)$. However, the percentage of apoptotic cells was higher than that of necrotic cells under all conditions.

\section{Localization of PS I in HeLa cells}

As displayed in Figure 3, diffuse cytoplasmic red staining was detected, indicating that after entry into cells, PS I localized to the cytoplasm around the nucleus of HeLa cells. No accumulation of PS I was observed inside the nucleus. Meanwhile, the merged stained images and fluorescence intensity profiles revealed that the red fluorescent signal of PS I exhibited a perfect overlap with the green fluorescent signal of mitochondria and the blue fluorescent signal of ER, 
A
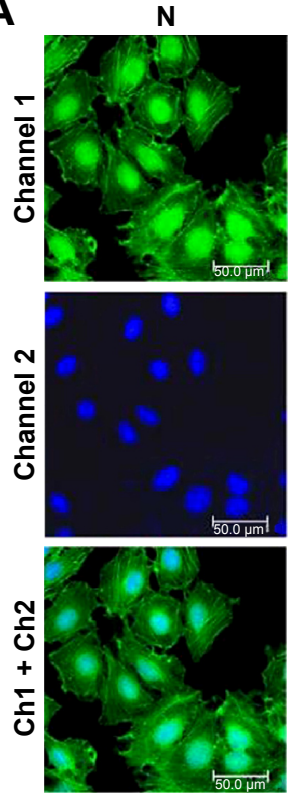

PDT
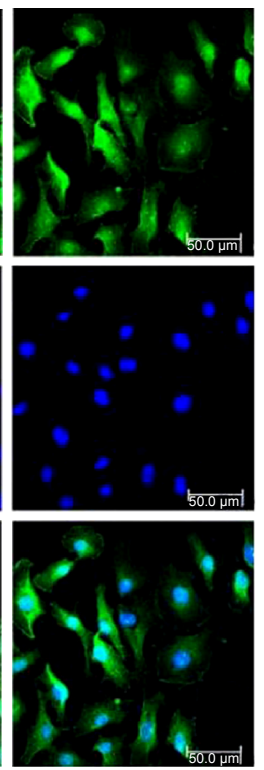

B
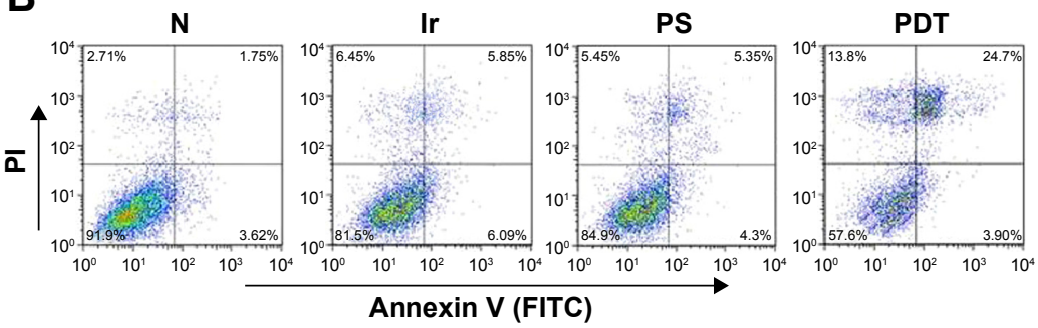

C

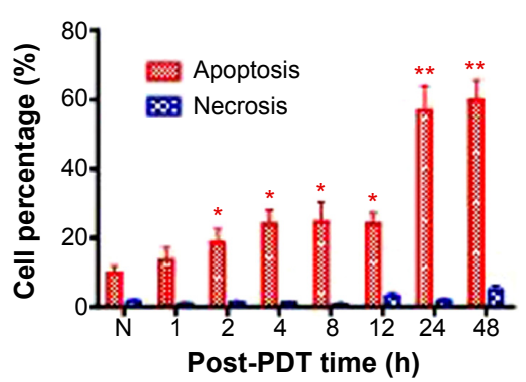

Figure 2 Changes in the cytoskeleton and DNA and apoptotic characteristics of HeLa cells after PDT.

Notes: (A) Changes in F-actin and DNA structures. (B) Results of flow cytometry analysis. N, untreated cells; Ir, only treated with $18 \mathrm{~J} / \mathrm{cm}^{2}$ of red light; PS, only incubated in $15.2 \mu \mathrm{M}$ of PS I for 12 hours; PDT, treated with $15.2 \mu \mathrm{M}$ of PS I for 12 hours and then irradiated with $18 \mathrm{~J} / \mathrm{cm}^{2}$ of red light. The apoptotic cells were measured at 24 hours postPDT. (C) Post-PDT time dependence. HeLa cells were incubated in $15.2 \mu \mathrm{M}$ of PS I for 12 hours and then irradiated with $18 \mathrm{~J} / \mathrm{cm}^{2}$ of red light. The apoptotic cells were measured via flow cytometry analysis at the indicated times. (D) Irradiation dose dependence and concentration dependence of PDT. HeLa cells were incubated in I5.2 $\mu$ M of PS I for 12 hours; they were then either irradiated with the indicated dose of red light (red lines) or were incubated in the indicated concentration of PS I for 12 hours and then irradiated with $18 \mathrm{~J} / \mathrm{cm}^{2}$ of red light (blue lines). The apoptotic cells were measured at 24 hours post-PDT. Data represent means $\pm S D s(n=3)$. $* P<0.05$ vs control; $* * P<0.0$ I vs control. Abbreviations: FITC, fluorescein isothiocyanate; Ir, irradiation; PDT, photodynamic therapy; PI, propidium iodide; PS I, photosensitizer I; SD, standard deviation.
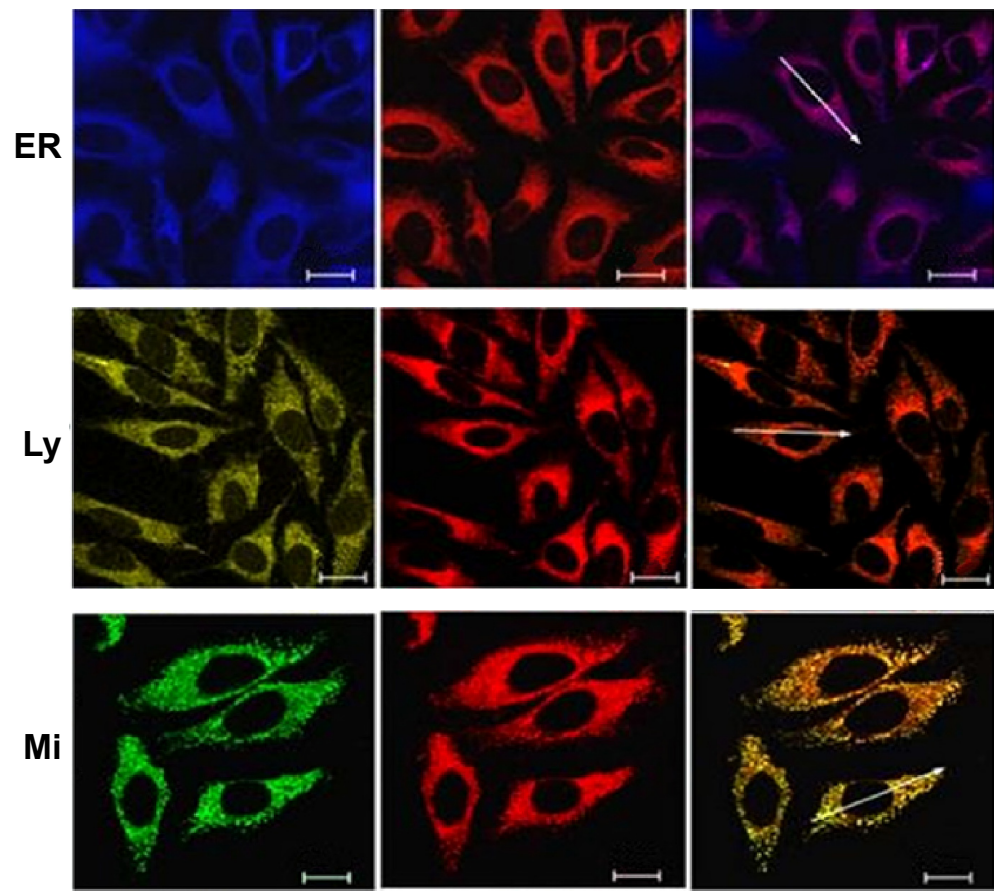

Probes

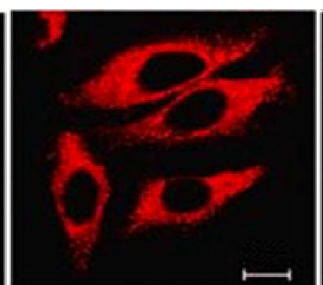

PS I
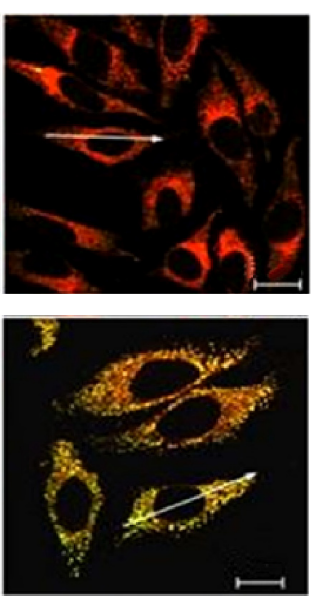

Merged
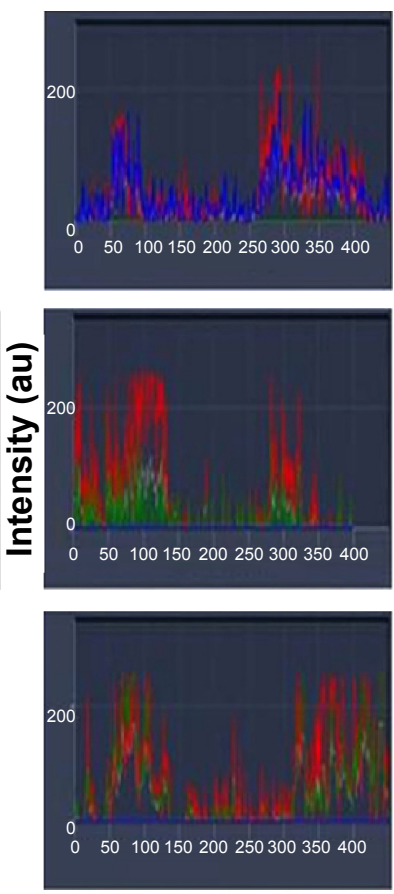

Distance (pixel)

Figure 3 Subcellular localization of PS I.

Notes: HeLa cells were incubated in PS I (I5.2 $\mu \mathrm{M}, 24$ hours) at $37^{\circ} \mathrm{C}$ and then Mito Tracker Green FM, LysoTracker Yellow HCK-I23, and ER-Tracker Blue-White DPX organelle probes were included during the final 0.5 hour. The cells were washed with DPBS prior to CLSM measurement. The first, second, and third rows represent the fluorescence of the organelle probes, PS I, and merge, respectively (left). The fluorescence intensity profiles of PS I (red lines) and the organelle probe (blue and green lines) were examined along the arrows in the confocal images (right). Images are representative of those obtained from three independent experiments. Scale bar, $20 \mu \mathrm{m}$.

Abbreviations: CLSM, confocal laser scanning microscopy; DPBS, Dulbecco's phosphate buffered saline; ER, endoplasmic reticulum; Ly, lysosomes; Mi, mitochondria; PDT, photodynamic therapy; PI, propidium iodide; PS I, photosensitizer I. 
but only a partial overlap with the yellow fluorescent signal of lysosomes, suggesting that both mitochondria and ER of the HeLa cells are the preferential sites of intracellular accumulation of PS I after 24-hour incubation.

\section{Evaluation of the levels of intracellular ROS and $\mathrm{Ca}^{2+}$}

The production of intracellular ROS was measured via the conversion of nonfluorescent $\mathrm{H}_{2}$ DCFDA to fluorescent DCFDA. As illustrated in Figure 4A, the generation of ROS was time dependent, with a maximum level threefold greater than that of the control cells at 20 minutes after PDT, which then gradually decreased. However, no clear changes in the generation of ROS were observed when HeLa cells were treated with PS I or irradiation alone.

Similarly, the level of intracellular $\mathrm{Ca}^{2+}$ rapidly increased over time in the PS I PDT group and then reached a plateau (approximately threefold greater than that in the control cells; $P<0.01)$ at 15 minutes. However, within the tested time, irradiation treatment alone did not induce any significant changes in $\left[\mathrm{Ca}^{2+}\right]_{\mathrm{i}}$, and incubation with PS I only induced a slight increase $(P>0.05$ vs normal control) (Figure 4B).

\section{Effect of PS I PDT treatment on MMP}

Due to leakage of the dye from the mitochondria to the cytosol, the fluorescent signals of mitochondria stained using R123 distinctly weakened over time in the PDT group (Figure 4C), and the intensity of the R123 signal became very faint and diffuse at 2 hours post-PDT (Figure 4D), indicating mitochondrial depolarization. However, the slight reduction in the fluorescence intensity of R123 over time in the other three groups may be due to self-quenching of the fluorescence (Figure 4C).
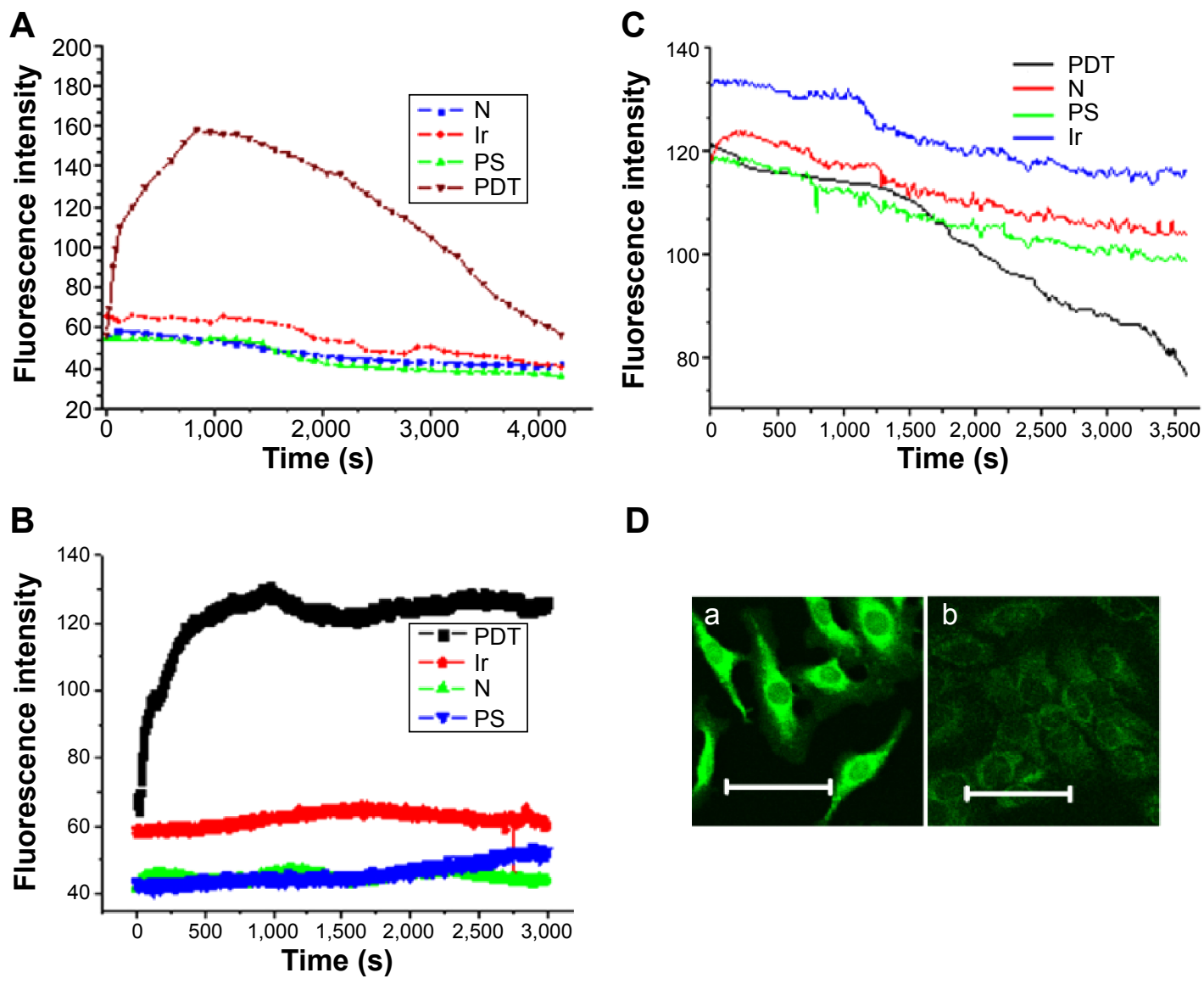

D

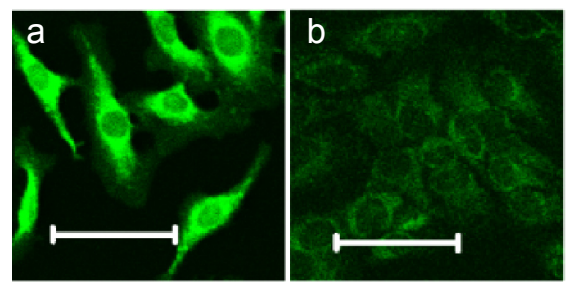

Figure 4 Alterations in intracellular (A) ROS, (B) Ca ${ }^{2+}$ levels, and (C) MMP and (D) confocal images of HeLa cells stained with RI23.

Notes: (A-C) Approximately 30 cells were randomly selected from three independent experiments, and fluorescence intensities were determined by processing the fluorescence images at a single-cell level. Intracellular ROS and $\mathrm{Ca}^{2+}$ levels, as well as the MMP, were measured via continuous confocal scanning. $\mathrm{N}$, untreated cells; $\mathrm{Ir}$, only treated with I8 $/ \mathrm{cm}^{2}$ of red light; PS, only incubated in I5.2 $\mu$ M of PS I for I 2 hours; PDT, incubated in I5.2 $\mu$ M of PS I for I 2 hours and then irradiated with I 8 J/cm² of red light. (D) Confocal images of HeLa cells stained with RI23. Cells were incubated in I5.2 $\mu$ M of PS I for I 2 hours and coincubated with RI 23 for the final half hour; cells were then irradiated with I 8 J/cm ${ }^{2}$ of red light. The fluorescence of RI23 was measured (a) soon after PDT or (b) 2 hours post-PDT. Scale bar, $80 \mu \mathrm{m}$.

Abbreviations: Ir, irradiation; MMP, mitochondrial membrane potential; PDT, photodynamic therapy; PS I, photosensitizer I; RI23, rhodamine I23; ROS, reactive oxygen species. 


\section{Apoptosis signaling pathways induced by PS I PDT}

As shown in Figure 5A and B, irradiation treatment alone or incubation in PS I without light resulted in the downregulation of $\mathrm{Bcl}-2$, the upregulation of $\mathrm{Bax}$, the induction of CHOP and GRP78, and the activation of caspase-12 and caspase-3; but this treatment had little effect on the release of cytochrome $\mathrm{c}$ and the activation of caspase-9. However, the release of cytochrome $\mathrm{c}$ induced by PS I PDT began as early as 1 hour post-PDT, then gradually increased and reached a peak at 8 hours after PDT (threefold greater than the level in control cells). The downregulation of Bcl-2 and the upregulation of Bax induced by PS I PDT were much stronger, which began dramatically at 2 hours after PDT and were sustained up to 48 hours after PDT. Meanwhile, the expression of caspase-12, CHOP, and GRP78, three proteins related to ER stress, was clearly detected at 1 hour post-PDT and these proteins exhibited an expression pattern of an early rise followed by a decrease. Moreover, activation of both caspase- 9 and caspase- 3 was apparently enhanced in the PDT group. Interestingly, the expression of caspase- 9 and caspase-3 shared a similar pattern: an increase soon after PDT, which continued up to 48 hours post-PDT. However, the level of caspase-9 expression was consistently higher than the level of caspase-3 expression throughout the experiment.

\section{Discussion}

Chlorin-type photosensitizers, due to their photophysical properties, are a topic of intensive research. Although mTHPC was approved by the US Food and Drug Administration for clinical applications, it also suffers from some drawbacks, such as limited tumor targeting and unsatisfactory solubility. A new photosensitizer - PS I - prepared in our laboratory by linking an analog of mTHPC to folic acid via PEG, displayed improved tumor targeting and increased aqueous solubility compared to the precursor, chlorin. ${ }^{8}$ The photocytotoxicity of PS I was also confirmed in the present study. Neither irradiation alone nor PS I without light was cytotoxic to HeLa cells, but PS I PDT displayed significant photocytotoxicity. This result is consistent with the changes in intracellular ROS and $\left[\mathrm{Ca}^{2+}\right]$. PS I PDT could result in a rapid and significant production of ROS and an overload of intracellular $\mathrm{Ca}^{2+}$, but irradiation treatment alone or incubation in PS I without light could not (Figure 4A and B). These results indicate that the decrease in viability of HeLa cells after PDT is due to the generation of intracellular ROS and $\left[\mathrm{Ca}^{2+}\right]_{\mathrm{i}}$ overload.

Because the activation of cell death pathways is largely dependent on the photosensitizer characteristics, oxygen concentration, and intensity of light, as well as the cell type, the definitive cell death mechanisms induced by PDT have not been fully elucidated. ${ }^{12}$ mTHPC was reported to be primarily localized to the membranes of the ER and the Golgi apparatus, and apoptosis has been reported as the principal mode of cell death mediated by mTHPC PDT. ${ }^{13,14}$ However, in this study, PS I was found to primarily reside in the mitochondria as well as the ER after endocytosis. Apoptosis remained the principal mode of HeLa cell death induced by PS I PDT, and cytotoxicity occurred in a manner dependent on concentration, irradiation dose, and time. Because of their size, macromolecular conjugates have been reported to be capable of being absorbed into cells only via endocytosis. ${ }^{15}$ In this case, except for passive
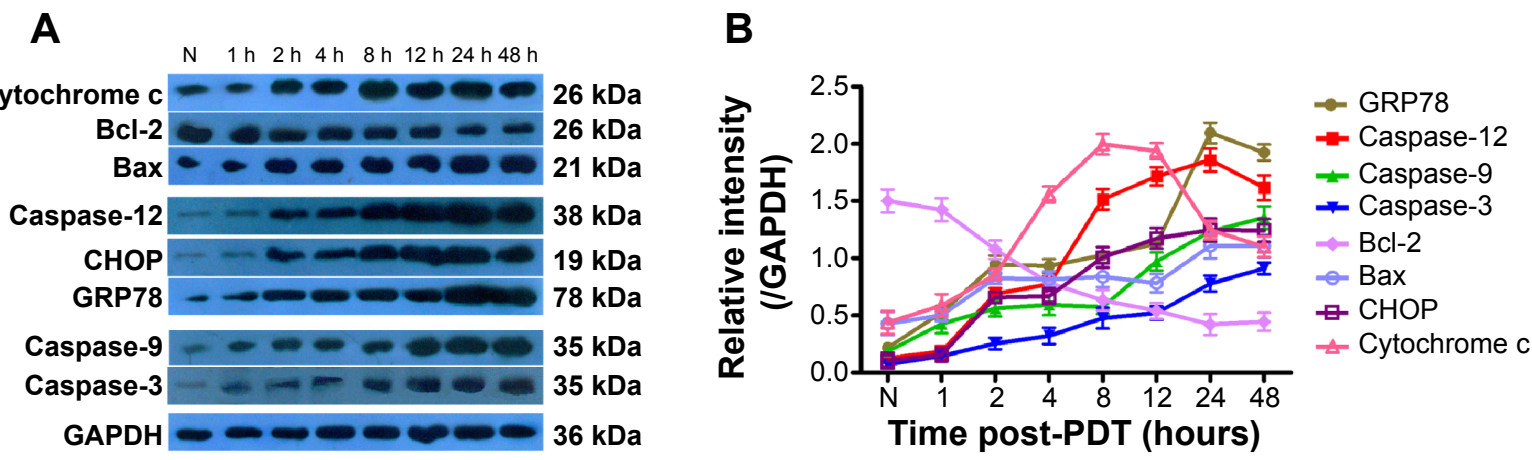

Figure 5 PS I PDT-induced expression of cytochrome c, CHOP, Bax, Bcl-2, caspase-3, caspase-9, caspase-12, and GRP78 in HeLa cells.

Notes: (A) Western blot analysis following PDT. HeLa cells were incubated in $15.2 \mu$ M of PS I for 12 hours and then exposed to $18 \mathrm{~J} / \mathrm{cm}^{2}$ of red light. Following cell harvesting at I hour, 2 hours, 4 hours, 8 hours, I 2 hours, 24 hours, and 48 hours after PDT, samples containing equal amounts of protein ( $100 \mu g$ ) were loaded into each lane of a sodium dodecyl sulfate-polyacrylamide gel. The protein bands were detected using a Kodak in vivo image analyzer. Protein bands in the grayscale images were quantified using Glyko Bandscan software (Glyko, Novato, CA, USA). GAPDH was used as a loading control. A representative trial of three independent experiments is presented. (B) Quantitative analysis of protein expression according to panel $(\mathbf{A})$. The vertical axis represents the protein expression relative to $\mathrm{GAPDH}$. Data represent means $\pm \mathrm{SDs}(\mathrm{n}=3$ ).

Abbreviations: CHOP, C/EBP homologous protein; GAPDH, glyceraldehyde-3-phosphate dehydrogenase; GRP, glucose-regulated protein; PDT, photodynamic therapy; PS I, photosensitizer I. 
endocytosis, there was likely active endocytosis mediated by the folate receptor. ${ }^{8,16}$ It is reasonable that the lipophilic photosensitizer tends to accumulate in membrane organelles such as mitochondria or lysosomes. On the other hand, other reports indicate that the aggregation state of intracellular photosensitizers affects their localization, with more aggregationprone species tending toward lysosomal localization. ${ }^{17}$ Due to the amphiphilicity of the PEG linker, a clear decrease in the aggregation of PS I has been observed previously. ${ }^{8}$ Therefore, we hypothesize that PEGylation leads PS I to localize more at the mitochondria and the ER than at the lysosomes.

The fact that PS I was primarily localized at the mitochondria and the ER prompted us to further examine mitochondrial and ER stress-associated apoptosis. There is increasing evidence that mitochondria play a critical role in the apoptotic cascade, and the release of cytochrome c from the mitochondria into the cytosol is a hallmark of cells undergoing apoptosis. ${ }^{18}$ In the present study, a rapid loss of MMP was observed after PDT, which was followed by the time-dependent release of cytochrome c. The opening of mitochondrial membrane permeability transition pores, which results in the dissipation of the MMP, has been proposed as the principal mechanism for the release of cytochrome c. ${ }^{19}$ Accumulating evidence suggests that Bcl-2, residing in the mitochondrial outer membrane and the ER membrane, performs the unique functional role of antiapoptosis via stabilization of the mitochondrial membrane..$^{20,21}$ PS I PDT induced an almost twofold and time-dependent downregulation in Bcl-2 protein expression and a marked increase in the expression of Bax, a proapoptotic protein. During apoptosis, Bax oligomerizes in the mitochondrial outer membrane and likely disrupts its integrity, freeing proapoptotic proteins such as cytochrome c, which allows for activation of caspase-9.22

Otherwise, intracellular $\mathrm{Ca}^{2+}$ overload attracted our attention. Intracellular $\mathrm{Ca}^{2+}$ overload may be due to the influx of extracellular $\mathrm{Ca}^{2+}$ or the release of $\mathrm{Ca}^{2+}$ from different intracellular stores (eg, the ER, the primary intracellular $\mathrm{Ca}^{2+}$ store) following photoinduced injury. ${ }^{9}$ ER stress-induced apoptotic cell death represents a novel mitochondrial-independent intrinsic apoptotic pathway. ${ }^{23,24}$ To specify the effect of ER stress on HeLa cell apoptosis induced by PS I PDT, the expression levels of caspase-12, CHOP, and GRP78 were monitored via Western blot analysis. Caspase- 12 is an important caspase involved in ER-induced apoptosis. ${ }^{25} \mathrm{CHOP}$ is an ER-stress-inducible transcription factor and is considered a major trigger of ER-stress-induced apoptosis by reducing the expression of Bcl-2. ${ }^{26}$ GRP78 is an ER chaperone and a key regulator of ER stress response signaling ${ }^{27}$ In our study, the activation of caspase-12 and the induction of CHOP and GRP78 shared the same response pattern, namely, an early increase followed by a decrease, as oxidative stress is induced by PS I PDT. Additionally, as CHOP was induced, the level of Bcl-2 expression was reduced following PDT (Figure 5A and B). All of these results probably reflect the initiation of the ER-stress-specific caspase cascade of apoptosis. Interestingly, although both irradiation treatment alone and incubation in PS I without light could not induce the generation of ROS or $\left[\mathrm{Ca}^{2+}\right]_{i}$ overload, they induced the ER stress response to some extent through the activation of caspase-12 and the induction of CHOP and GRP78.

Activation of both caspase- 9 and caspase-3 were observed soon after PDT, suggesting that PS I PDT may induce apoptotic cell death via a caspase-dependent pathway. However, it is worth noting that the level of caspase- 9 expression was consistently higher than that of caspase- 3 throughout our experiments (Figure 5A and B). It is well known that caspase- 9 is an initiator caspase of apoptosis and can be activated by mitochondrial events and ER stress, ${ }^{28}$ but caspase- 3 is a downstream substrate of caspase- 9 and a key player in the execution phase of apoptosis. Thus, the activation of caspase- 9 should induce the activation of caspase- 3 . Therefore, we hypothesize that the difference in expression of caspase- 9 and caspase- 3 could be due to the induction of GRP78, as some reports have indicated that overexpression of GRP78 could reduce apoptosis and mitigate cytosolic $\mathrm{Ca}^{2+}$ overload induced by ER stressors in some cell types. ${ }^{29}$

\section{Conclusion}

In summary, after active endocytosis mediated by the folate receptor, PS I is principally localized at the mitochondria and the ER of HeLa cells. Apoptosis is the primarily preferred mechanism of HeLa cell death in our system. The rapid generation of ROS in HeLa cells following PDT provoked mitochondrial damage and ER stress, which, in turn, activated the intrinsic apoptotic pathway. Specifically, compared to $\mathrm{mTHPC}$, after the structural modifications reported by our research group, the mitochondria became the primary site of PS I localization and the targeting of PS I for folate receptor-positive cells was obviously improved; however, the cell death mode induced by PS I PDT was similar to that induced by mTHPC PDT.

\section{Acknowledgment}

We are grateful for the financial support from the National Natural Science Foundation of China (project number 21072227). 


\section{Disclosure}

The authors report no conflicts of interest in this work.

\section{References}

1. Song J, Chen Q, Xing D. Enhanced apoptotic effects by downregulating Mcl-1: evidence for the improvement of photodynamic therapy with celecoxib. Exp Cell Res. 2013;19:1491-1504.

2. Gijsens A, Missiaen L, Merlevede W, de Witte P. Epidermal growth factor-mediated targeting of chlorin e6 selectively potentiates its photodynamic activity. Cancer Res. 2000;60:2197-2202.

3. St Denis TG, Hamblin MR. Synthesis, bioanalysis and biodistribution of photosensitizer conjugates for photodynamic therapy. Bioanalysis. 2013;5:1099-1114.

4. Stefflova K, Li H, Chen J, Zheng G. Peptide-based pharmacomodulation of a cancer-targeted optical imaging and photodynamic therapy agent. Bioconjug Chem. 2007;18:379-388.

5. Hu Z, Rao B, Chen S, Duanmu J. Targeting tissue factor on tumor cells and angiogenic vascular endothelial cells by factor VII-targeted verteporfin photodynamic therapy for breast cancer in vitro and in vivo in mice. BMC Cancer. 2010;10:235-248.

6. McCarthy JR, Bhaumik J, Merbouh N, Weissleder R. High-yielding syntheses of hydrophilic, conjugatable chlorins and bacteriochlorins. Org Biomol Chem. 2009;7:3430-3436.

7. Vlahov IR, Leamon CP. Engineering folate-drug conjugates to target cancer: from chemistry to clinic. Bioconjug Chem. 2012;23: 1357-1369.

8. Li D, Li P, Lin H, Jiang Z, Guo L, Li B. A novel chlorin-PEG-folate conjugate with higher water solubility, lower cytotoxicity, better tumor targeting and photodynamic activity. J Photochem Photobiol B. 2013; 127:28-37.

9. Zhang Q, Xiang G, Zhang Y, et al. Increase of doxorubicin sensitivity for folate receptor positive cells when given as the prodrug $\mathrm{N}$-(phenylacetyl)doxorubicin in combination with folate-conjugated PGA. J Pharm Sci. 2006;95:2266-2275.

10. Buytaert E, Dewaele M, Agostinis P. Molecular effectors of multiple cell death pathways initiated by photodynamic therapy. Biochim Biophys Acta. 2007;1776:86-107.

11. Szurko A, Rams M, Sochanik A. Spectroscopic and biological studies of a novel synthetic chlorin derivative with prospects for use in PDT. Bioorg Med Chem. 2009;17:8197-8205.

12. Robertson CA, Evans DH, Abrahamse H. Photodynamic therapy (PDT): a short review on cellular mechanisms and cancer research applications for PDT. J Photochem Photobiol B. 2009;96:1-8.

13. Teiten MH, Bezdetnaya L, Morlière P, Santus R, Guillemin F. Endoplasmic reticulum and Golgi apparatus are the preferential sites of Foscan 1 localization in cultured tumor cells. Br J Cancer. 2003;88:146-152.
14. Senge MO, Brandt JC. Temoporfin (Foscan, 5,1015,20-Tetra (m-hydroxyphenyl)chlorin) - a second-generation photosensitizer. Photochem Photobiol. 2011:87:1240-1296.

15. Duncan R, Spreafico F. Polymer conjugates. Pharmacokinetic considerations for design and development. Clin Pharmacokinet. 1994;27: 290-306.

16. Low PS, Kularatne SA. Folate-targeted therapeutic and imaging agents for cancer. Curr Opin Chem Biol. 2009;13:256-262.

17. Hamblin MR, Miller JL, Rizvi I, Ortel B, Maytin EV, Hasan T. Pegylation of a chlorine6 polymer conjugate increases tumor targeting of photosensitizer. Cancer Res. 2001;61:7155-7162.

18. Zamzami N, Kroemer G. Apoptosis: mitochondrial membrane permeabilization - the whole story? Curr Biol. 2003;13:R71-R73.

19. Mroz P, Yaroslavsky A, Kharkwal GB, Hamblin MR. Cell death pathways in photodynamic therapy of cancer. Cancer. 2011;3:2516-2539.

20. He GF, Bian ML, Zhao YW, Xiang Q, Li HY, Xiao C. A study on the mechanism of 5-aminolevulinic acid photodynamic therapy in vitro and in vivo in cervical cancer. Oncol Rep. 2009;21:861-868.

21. Chen X, Zhao P, Chen F, Li L, Luo R. Effect and mechanism of 5 -aminolevulinic acid-mediated photodynamic therapy in esophageal cancer. Lasers Med Sci. 2011;26:69-78.

22. Panzarini E, Inguscio V, Dini L. Timing the multiple cell death pathways initiated by rose Bengal acetate photodynamic therapy. Cell Death Dis. 2011;2:e169.

23. Zhou Z, Yang H, Zhang Z. Role of calcium in phototoxicity of 2-butylamino-2-demethoxy-hypocrellin A to human gastric cancer MGC-803 cells. Biochim Biophys Acta. 2003;1593:191-200.

24. Rao RV, Castro-Obregon S, Frankowski H, et al. Coupling endoplasmic reticulum stress to the cell death program. An Apaf-1-independent intrinsic pathway. J Biol Chem. 2002;277:21836-21842.

25. Chung PS, He P, Shin JI, Hwang HJ, Lee SJ, Ahn JC. Photodynamic therapy with 9-hydroxypheophorbide $\alpha$ on AMC-HN-3 human head and neck cancer cells. Cancer Biol Ther. 2009;8:1343-1351.

26. Szegezdi E, Logue SE, Gorman AM, Samali A. Mediators of endoplasmic reticulum stress-induced apoptosis. EMBO Rep. 2006;7: $880-885$.

27. Firczuk M, Gabrysiak M, Barankiewicz J, et al. GRP78-targeting subtilase cytotoxin sensitizes cancer cells to photodynamic therapy. Cell Death Dis. 2013;4:e741

28. Morishima N, Nakanishi K, Takenouchi H, Shibata T, Yasuhiko Y. An endoplasmic reticulum stress-specific caspase cascade in apoptosis. Chtochrome c-independent activation of caspase- 9 bycaspase- 12 . J Bio Chem. 2002;277:34287-34294.

29. He P, Ahn JC, Shin JI, Chung PS. Photoactivation of 9-hydroxypheophorbide $\alpha$ triggers apoptosis through the reactive oxygen speciesmediated mitochondrial pathway and endoplasmic reticulum stress in AMC-HN-3 laryngeal cancer cells. Int J Oncol. 2010;36:801-808.
OncoTargets and Therapy

\section{Publish your work in this journal}

OncoTargets and Therapy is an international, peer-reviewed, open access journal focusing on the pathological basis of all cancers, potential targets for therapy and treatment protocols employed to improve the management of cancer patients. The journal also focuses on the impact of management programs and new therapeutic agents and protocols on

\section{Dovepress}

patient perspectives such as quality of life, adherence and satisfaction. The manuscript management system is completely online and includes a very quick and fair peer-review system, which is all easy to use. Visit http://www.dovepress.com/testimonials.php to read real quotes from published authors. 